Acta Technologica Agriculturae 3

Nitra, Slovaca Universitas Agriculturae Nitriae, 2014, pp. 70-74

\title{
RESEARCH METHODS FOR TRIBOLOGICAL PROPERTIES OF RESTORATIVE AND PREVENTIVE COATINGS IN DIFFERENT LUBRICATING MEDIA AT SLIDING FRICTION
}

\author{
Mitko NIKOLOV*, Plamen KANGALOV \\ 'Angel Kanchev' University of Ruse, Bulgaria
}

A large part of machines (85-90\%) (Garkunov, 2003) lose their efficiency due to wear of parts, which disrupts their normal interaction, causes additional loading, shocks and vibrations, leads to seizures and jams and to accidents in many cases. This paper presents research methods for tribological properties of restorative and preventive coatings in different lubricating media at sliding friction.

Keywords: tribological research, research model of refurbishing surface, research method, parameters of physical model of tribological research

A large part of machines (85-90\%) (Garkunov, 2003) lose their efficiency due to wear of parts, which disrupts their normal interaction, causes additional loading, shocks and vibrations, leads to seizures and jams and to accidents in many cases. Even an insignificant wear of working surfaces of parts not only reduces the output parameters of machines but often causes them to stop and creates significant downtime.

The running-in of friction compounds, which occurs in the final stage of making, restoration and repair or in the initial stage of machine exploitation, determines their flawlessness and durability to a great extent. Wear in the process of running-in has an immediate impact on the durability of compounds as this wear limits the range of work change of friction parts dimensions in the process of operation. The enhancement of wearing qualities of restored and newly made parts is of great importance for the economy of every country as it decreases the costs for spare parts, raw materials, materials and energy.

The aim of research methods is to experimentally establish the main tribological properties of running-in and wear of friction surfaces with restorative and preventive coatings during sliding friction in different lubricating media.

The object of research methods are lubricating media, weld overlaid and galvanic coatings, and the subject of research are their tribological properties.

\section{Exposition}

Tribological research methods include a choice of cybernetic and physical models of research, mode of friction and wear, methods and means for measuring process parameters, data processing and presentation of obtained results. Research methods and the cybernetic model are developed in accordance with the requirements for friction and wear in conditions that correspond to operational capacities and those of technical means for these studies. One of the main constituents of study is its cybernetic model. The model for study according to cybernetic principles is defined with the main input factors and output parameters of the object of study (Figure 1). The input factors chosen for the cybernetic model of study are: the parameters of qualities of friction surfaces materials; the parameters of loading of the friction pair; the parameters of properties of the friction and wear environment; the parameters of duration of testing experiments of the friction pair (Mashkov et al., 2000; Nikolov et al., 2003; Tonchev and Kangalov, 2003). The main output parameters of the model for study are the vectors of wear parameters (dimensions, dynamics, speed, intensity, wearing qualities of the friction pair and its elements); time of running-in and established wear; friction torque at loading, running-in and wear; roughness and micro-hardness of friction surfaces. From the output parameters, the main criterion for evaluation of the running-in and wear of restorative and preventive

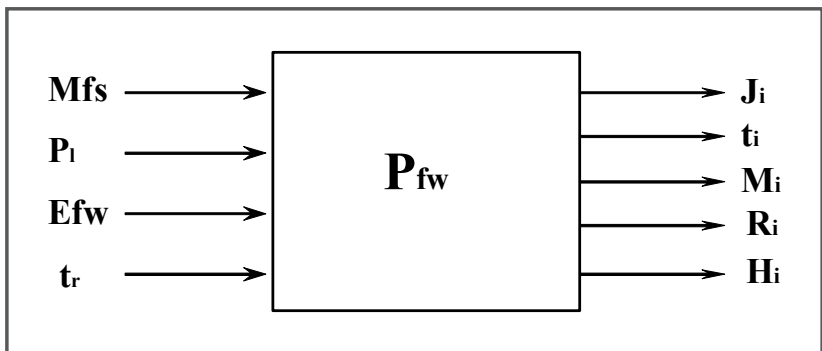

Figure 1 Cybernetic model for tribological research of restorative and preventive coatings during sliding friction

Mfs - parameters of properties of friction surfaces materials; $P_{1}$ - parameters of loading of the friction pair; Efw - parameters of properties of the friction and wear environment; $t$ - parameters of the time of testing experiments of the friction pair; $J_{i}$ - vector of wear parameters; $\mathrm{t}$ - time for running-in; $\mathrm{M}_{\mathrm{i}}$ - friction torque at loading, running-in and wear and testing; $R_{-}$- roughness of the friction surface; $H_{-}-$hardness of the friction surface) 
coatings is the vector of wear parameters (Nikolov et al., 2003; Tonchev and Kangalov, 2003).

The auxiliary criteria for evaluation of tribological properties of restorative and preventive coatings are: the time for running-in and established wear of the friction pair; the parameters of friction torque at loading, running-in and established wear; the parameters of the roughness of friction surfaces and coatings in the beginning, at their running-in and established wear; the parameters of the micro-hardness of friction surfaces and coatings in the beginning, at their running-in and established wear.

Apart from the cybernetic model, the tribological research of properties of restorative and preventive coatings includes also a physical model (Figure 2) for research and related methods and means for evaluation of parameters of the process of running-in and wear of the friction pair (Nikolov et al., 2003; Tonchev and Kangalov, 2003).

The parameters of the physical model are chosen on the basis of structural properties of new and worn parts. The structure of the physical model for tribological research is expressed by parameters of the friction pair. The main signs of the model for study are: the relative motion of friction surfaces; the scheme and environment of friction and the types of wear, which are defined by the capacity of the machine for friction and wear and its accessories, systems and devices.

A great number of restored and worn parts are damaged from sliding friction and hence the physical model of the friction pair shaft-bearing is the pair roller-sector with respective parameters of geometrical and physical similarity (Figure 2).

In accordance with the environment, chosen conditions and modes of friction, wear can be mechanical, molecularmechanical or corrosion-mechanical and has to correspond to the nature of wear of parts in operating conditions. The friction and wear environment of tested samples is determined by conditions for liquid and limit friction with different motor and transmission oils, the parameters of which are standard and widely used in the process of development and operation in the tractor and agricultural machinery. Friction modifiers of different type and concentration could be added to standard motor and transmission oils where corrosion-mechanical wear is dominant.

The parameters of the physical model of tribological research are of four types: geometrical, mechanical, physical and technological. These parameters are chosen based

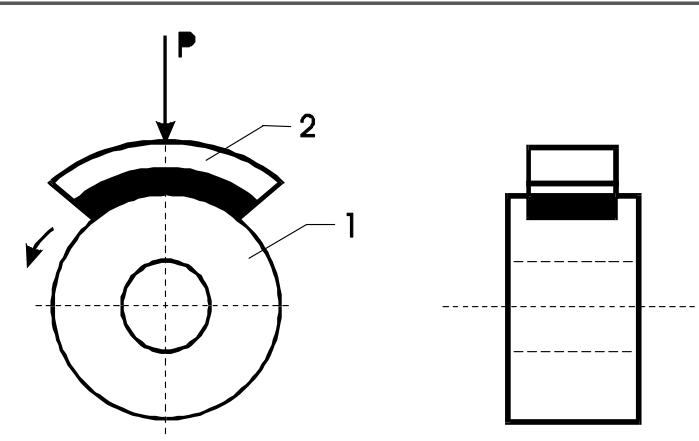

Figure 2 Physical model of the friction pair 1 - roller; 2 - sector on the statistical analysis of constructive- technological and operational characteristics of worn and restored parts (Tonchev and Stanev, 1979).

The geometrical parameters include the dimensions of the pair and the macro- and micro-geometry of friction surfaces. The dimensions of the pair are the diameter and width of the roller and sector as well as the clearance between them. Macro-geometry is defined by the unalignment and perpendicularity of the roller and inaccuracy of base surfaces of the sector of the pair, whereas micro-geometry is defined by the roughness of friction surfaces for the roller and sector.

The mechanical parameters include internal stresses, the macro- and micro-hardness of friction surfaces, the physical parameters - the mass of the body and counter body, and the technological parameters - the thickness of the coating and antifriction layer, the macro- and micro-structure of the surface layer of the roller and sector.

Master samples for testing the durability of restorative and preventive coatings are from the most widespread materials for sliding bearings in the tractor and agricultural machinery - steel 45 and leaded bronze БО - 30. Samples (rollers and sectors) from weld overlaid and electro-chemical coatings are put to comparative study, and also at a different composition of the lubricating medium.

The master and restored samples of rollers are processed thermally and mechanically. Their hardness is $55^{ \pm 2} \mathrm{HRC}$ $\left(\mathrm{HV}_{5}=6,100 \pm 400 \mathrm{MPa}\right)$, and they are polished to final size following a special method, after they have been used for the manufacturing of testing rollers. The rollers have a dimension of the friction surface of $50^{+0.02} \mathrm{~mm}$, thickness of $12^{+0.05} \mathrm{~mm}$, thickness of the weld overlaid coating of $0.5 \mathrm{~mm}$ along the radius of roughness $R_{a}=0.28-0.32 \mu \mathrm{m}$, and a mass of $160-170 \mathrm{~g}$.

The sectors of the friction pair are bimetal, with steel basis coated with an antifriction layer:

- from $5 \mathrm{O}-30$ with hardness $\mathrm{HV}_{5}=490 \pm 50 \mathrm{MPa}$;

- from electro-chemical alloy EO - 92 on the basis of lead from silicofluoride electrolyte, the composition of which is described by Kangalov (1994). The deposition of the electro-chemical coating is of a density of DC DK $=2 \mathrm{~A} / \mathrm{dm}^{2}$ at temperatures of $18-25^{\circ} \mathrm{C}$.

Master and restored sectors for friction with rollers were cut from steel rings prepared in advance, coated with an antifriction layer of $\mathrm{BO}-30$ with a thickness of $0.1-0.5 \mathrm{~mm}$ along radius and EO - 92 with a thickness of $0.05-0.25 \mathrm{~mm}$ along radius. Prior to cutting the bearing rings with a central angle of $46^{\circ}$, from which sectors are made, the outer surface is polished with a diameter of $70^{+0.1} \mathrm{~mm}$, and the inner surface of bearing rings is ground to a diameter of $50_{+0.04}^{+0.06} \mathrm{~mm}$ with a roughness of $R_{a}=2.0-2.8 \mu \mathrm{m}$. The clearance of the pair has to be $0.04 \mathrm{~mm}$ so that there could be formed a uniform oil wedge during friction. The length of the sector friction surface is $20 \mathrm{~mm}$, the width is $10^{+0.05}$, and the area of the support surface is $2 \mathrm{~cm}^{2}$ with a mass of $15-20 \mathrm{~g}$.

Closely related to the cybernetic model of tribological research methods is the mode for study of the friction pair where three types of parameters are defined: dynamic, kinematic and thermodynamic. Dynamic refers to the parameters of loading of the friction pair (size, step, speed and time for loading), kinematic refers to the speed of 
rotation and speed of sliding of friction surfaces, and thermodynamic refers to the temperature of the friction surface and the temperature of lubricating media.

The loading of the friction pair 'roller-sector' is done steplessly at a speed of loading of $1 \mathrm{MPa} / \mathrm{min}$ and loading time $5 \mathrm{~min}$, at a load of $100 \mathrm{daN}$ and an accuracy of measurement 1 daN, which provides a pressure of $5 \mathrm{MPa}$ (Tonchev and Stanev, 1983). This size is close to the limit one and could be borne by different materials and coatings without seizure.

The speed of sliding is another important parameter, influencing to a great extent the processes of running-in and the size of initial wear. Preliminary studies show that a suitable frequency of rotation of the roller is $540 \mathrm{~min}^{-1}$. At this frequency of rotation, a considerable and measurable wear of the roller and sector is reached for a relatively short period of time of 1-2 hr during running-in and 3-4 hr during established wear. An increase in frequency leads to a decrease in wear and increase in testing time due to better hydrodynamic lubrication of the pair. Testing is done at a frequency of rotation of $540 \mathrm{~min}^{-1}$, and for the roller diameter of $50 \mathrm{~mm}$ the speed of sliding measured is $85 \mathrm{~m} /$ min and tribotechnical property PV $=425 \mathrm{MPa} \cdot \mathrm{m} / \mathrm{min}$. These values are accepted in accordance with permitted limits for the loading of sliding bearings (Tonchev and Stanev, 1983).

The temperature of the lubricating medium in the process of running-in must be within range $30-40{ }^{\circ} \mathrm{C}$ and is maintained at established wear at $40^{\circ} \mathrm{C}$ with an accuracy of $1{ }^{\circ} \mathrm{C}$. These temperatures correspond to the mode of tractor engines at starting, when a considerable wear is recorded.

The study of running-in and wear of friction surfaces with restored and preventive coatings in liquid lubricating medium is carried out on a special installation, the general outline of which is shown in Figure 3. The installation consists of: a machine for friction and wear SMC-2; a system for continuous mixing of the oil; a system for cooling of the friction chamber and continuous maintenance of temperature set in it; an electronic system for measuring and recording of friction torque, the duration of loading, running-in and established wear; an electronic system for measuring and recording of temperature in the friction chamber and water volume for cooling the chamber; an electronic recording system for the total frequency of the roller and the duration of experiment, respectively with an accuracy of $1 \mathrm{~min}^{-1}$ and $1 \mathrm{~s}$.

During the testing time, the installation provides continuous mixing of the oil, cooling of the oil and continuous maintenance of set temperature, measuring the total frequency of rotation (roller trajectory) and the time of experiment.

Research methods provide for using the machine for friction and wear SMC-2, allowing for wear of the friction surface during sliding, rolling and rolling with slip along the scheme 'roller-sector' and 'roller-roller', which model sliding and rolling bearings, gears and other similar pairs. It is driven

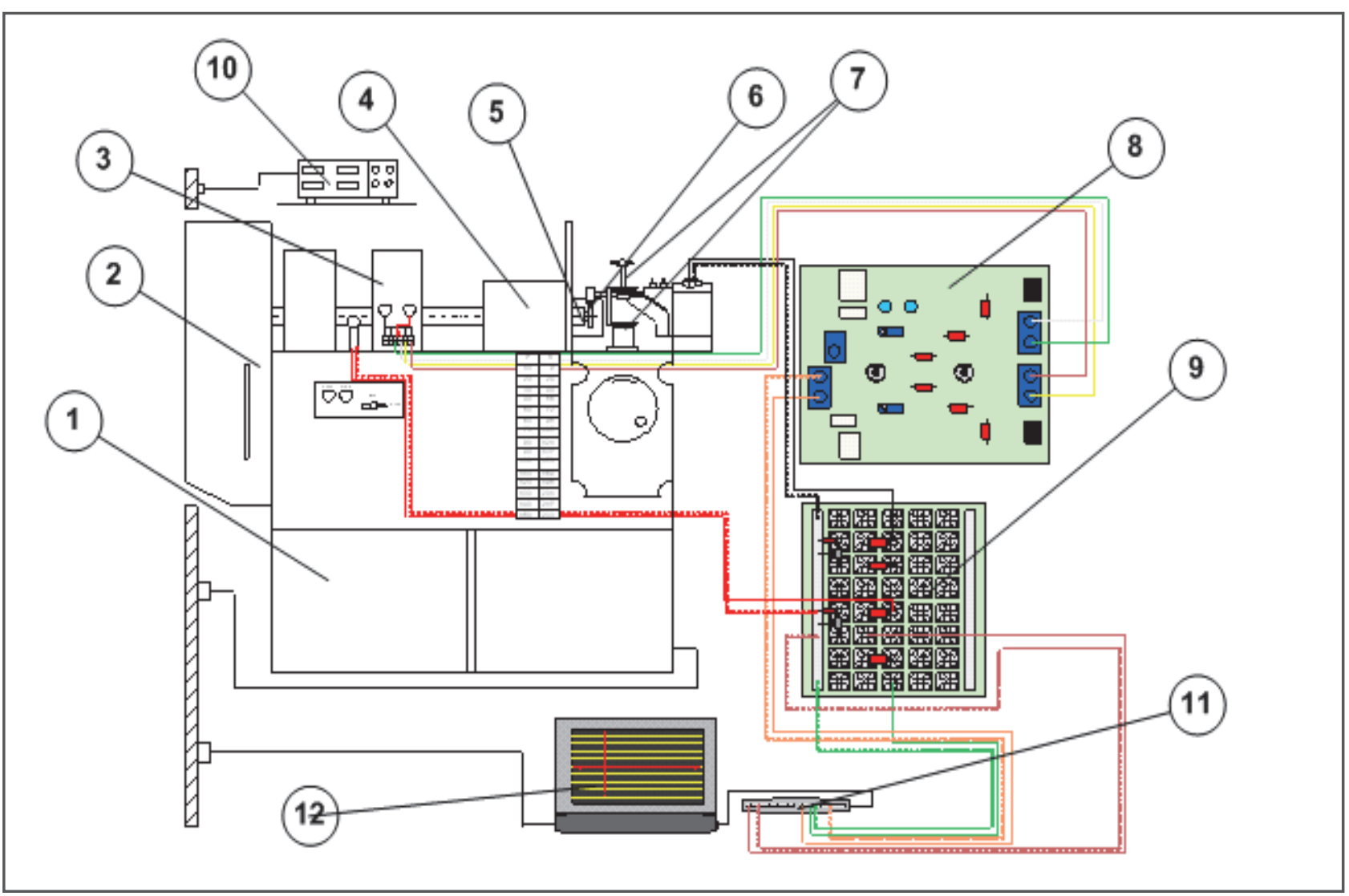

Figure 3 Installation scheme of tribological research for restorative and preventive coatings

1 - machine for friction and wear SMC-2; 2 - drive mechanism; 3 - rotation torque sensor; 4 - oil mixing system; 5 - water cooling chamber; 6 - tested pair, roller-sector'; 7 - loading system; 8 - friction torque differential circuit; 9 - shaft revolution circuit; 10 loading time pulse counter; 11 - multi-function product NI-USB 6210; 12 - portable computer (laptop)(b) 


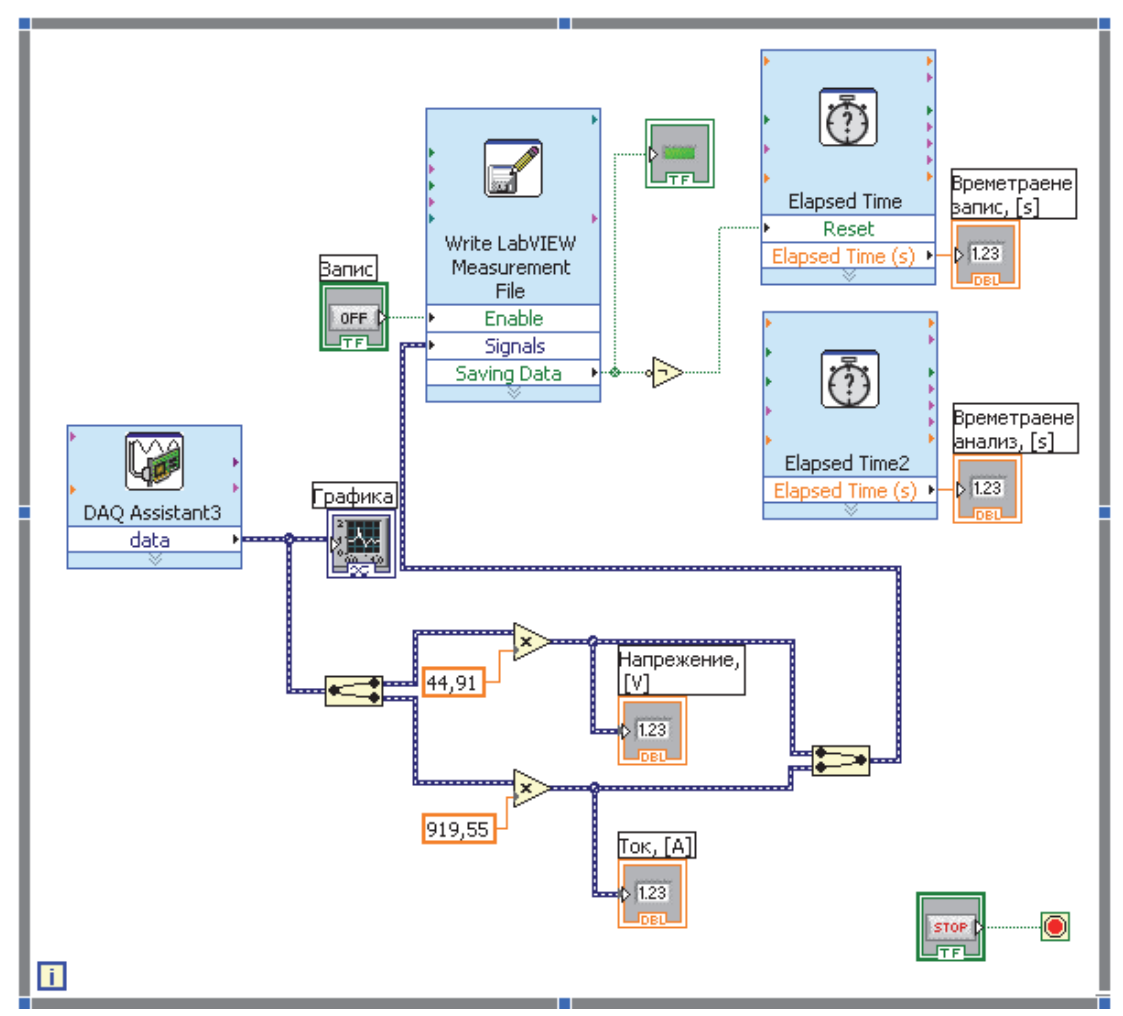

Figure 4

Flowchart for recording and conversion of friction torque

by a synchronous motor and has three degrees of rotation frequency: 300 , 540 and $1,000 \mathrm{~min}^{-1}$.

For the accurate measuring of tribological parameters, the machine for friction and wear SMC-2 is upgraded (Tonchev and Stanev, 1983) with various systems and devices to provide conditions for friction and wear close to operational: a small water cooling chamber with the capacity of lubricating medium of $150 \mathrm{ml}$; a device for calibration of instruments for measuring and recording of friction torque; a special master dynamometer pair loading; an indicating device for measuring the beating of the roller after the machine assembly with an accuracy of $0.01 \mathrm{~mm}$.

The loading of the friction pair is done with the help of a special spring and worm gear, and divisions are read on a graduated flywheel. The accuracy of one division is $0.70 \mathrm{~kg}$.

To maintain steady conditions of friction and wear, a special smallvolume water cooling chamber has for calibrating the device for friction

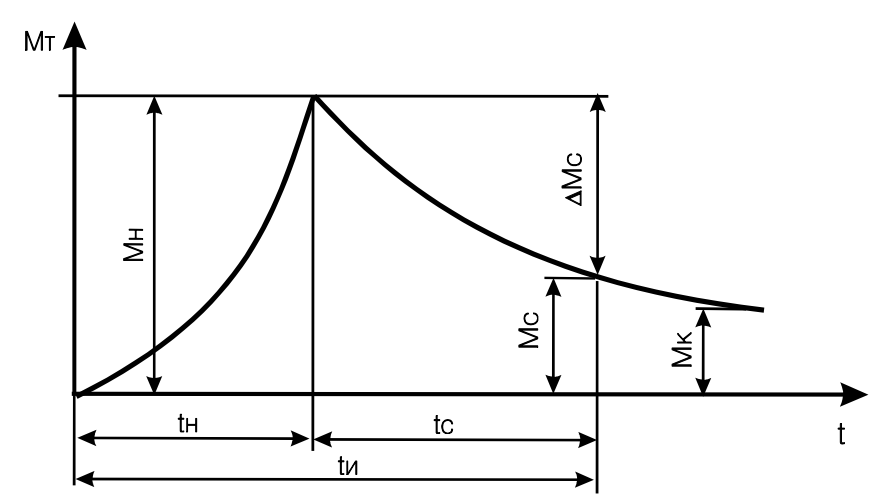

Figure 5 Dynamics of change in friction torque in the process of running-in and wear at stepless loading

$\mathrm{MH}_{\mathrm{H}}$ - friction torque at the end of loading; $\mathrm{Mc}$ - friction torque after running-in; $\Delta \mathrm{Mc}$ - friction torque during running-in; $\mathrm{MK}_{\mathrm{K}}$ - friction torque at the end of testing

been developed within the machine with the capacity of $150 \mathrm{ml}$ oil, for friction and wear at liquid and semi-liquid lubrication, allowing for continuous mixing of the oil. The oil mixing system is represented by air supply though a t-cock at the bottom part of the chamber, and the magnetic cleaning of wear products is done though four permanent magnets.

The system for cooling the chamber with oil serves for maintaining constant temperature during the testing of materials. It is a known fact that wear is greatly influenced by temperature during liquid lubrication. To maintain constant temperature in the chamber, a thermostat UTU - 4 is connected to the chamber cooling system.

The friction torque of the tested pair (Figure 5) is constantly measured and recorded with an inductive sensor, positioned between the gear and the spindle of the machine. For signal conversion from the inductive sensor, circuit 8 has been developed for friction torque (Figure 3), which determines the differential of two coils of the sensor. The reporting and recording of the signal coming out of the friction torque differential circuit is done with the help of the multi-function product NI-USB 6210. For the conversion of recorded data in N.cm, there is developed a flowchart on which stored data are recorded and visualised, embedded in the driving software NI-DAQmx and the software product Lab View (Figure 4). During the experimental tests, the device NI-USB 6210 is connected to the USB port of a portable computer, and data from recording the sliding friction process in real time are stored in a .xls document, and their subsequent processing is done using Microsoft Office Excel. Friction torque is continuously recorded during the testing time and is visualised on the personal computer screen in a digital and graphic format.

The total frequency of rotation of the roller is measured using the circuit converter 9 (Figure 3), which receives a signal for rotation frequency from the inductive sensor, which is mounted between the gear and the inductive sensor for measuring of friction torque. The signal from the circuit is measured, recorded and converted by the multifunction product NI-USB 6210, and 
the flowchart is shown in Figure 4. Current and total values of rotation frequency during the testing time are recorded and visualised on the personal computer screen in a digital format.

Experiments with friction surfaces of restored and preventive coatings are carried out until constant friction torque occurs. The friction torque the value of which does not change by above $1 \mathrm{Ncm}$ for a period of $10 \mathrm{~min}$ is assumed as constant. The time for active running-in is determined according to completing the steep section of the change of friction torque (Figure 5).

The size of wear is determined in accordance with the weight method divided for each of the friction parts (roller and sector), with the help of analytical scales WA - 33 with an accuracy of $0.05 \mathrm{mg}$, and all samples are cleaned with petrol, dried and weighed before and after experiment.

The roughness of friction surfaces before and after each test for running-in and wear is determined by one parameter $R_{a}$ in ten sections of the roller and in five sections of the sector, using a roughness measuring device Mitutoyo SJ-210. The device allows determining the size during the specific measuring, both in terms of $R_{a}$ and $R_{z}$.

The macro-roughness of friction surfaces is measured before the beginning of experiments according to Vickers method, with a hardness meter T -1 at loading of $5 \mathrm{~kg}$, and the micro-hardness of the roller and sector is measured before and after each test with a device ПMT - 3 at specific loading (100 $\mathrm{g}$ for the roller and $20-50 \mathrm{~g}$ for the sector in accordance with antifriction alloy). Preliminary measurements of the macro-hardness of rollers for selection are done using Rockwell method, scale ' $\mathrm{C}$ ', with a hardness meter TK -250 at a loading of $150 \mathrm{daN}$.

The study of running-in and wear of friction surfaces with restorative and preventive coatings in different lubricating media is done in the following sequence: preparation and calibration of the machine for friction and wear, systems, accessories and parts that go with it; preparation and measuring of samples for study; measuring the parameters of samples before and after each test; measuring and recording the input and output parameters of running-in and wear; processing of experimental data and presenting of final results analytically, in tables, and graphically.

To establish the dynamics of running-in and wear of tested preventive and restorative coatings, six experiments are carried out three times for each pair following the scheme: two one-hour-long; two two-hour-long, and two four-hour-long, and the total testing duration of one pair is $14 \mathrm{hr}$ (which comprises $448.10^{3} \mathrm{~m}$ friction trajectory). These structures of tests allow for a minimum time to cover and express the dynamics of change of output parameters of running-in and established wear of friction surfaces.

During the testing, analysis and interpretation of research results, the comparative and complex, system, cybernetic and physical research approaches, statistical methods and methods for single-factor and multi-factor planning of experiment are used. The results from measuring the observed parameters are recorded in files. Data are processed using Microsoft Office Excel by calculating the values of triblogial characteristics. The graphical relations between the input factors and output parameters of the process for running-in and wear of restorative and preventive coatings in different lubricating media are built based on obtained results.

\section{Conclusions}

1. Research methods of tribological characteristics have been suggested when lubricating media of restorative and preventive coatings are changed for parts from the tractor and agricultural machinery.

2. The size and speed of wear of the friction pair and its elements during running-in and established wear have been accepted as a main criterion for evaluating the running-in and wear of restorative and preventive coatings.

3. The auxiliary criteria for evaluation of tribological properties of restorative and preventive coatings are the following: the parameters of friction torque at loading, running-in and established wear; the parameters of the roughness of friction surfaces and coatings in the beginning, during their running-in and established wear; the time for running-in, established wear and testing of the friction pair; the parameters of the micro-hardness of the friction pair and coatings in the beginning, during their running-in and established wear.

\section{References}

GARKUNOV, D.N. 2003. Tribotechnology - construction, manufacturing and operation of machinery. M. Publishing house MSHA. 2003.

KANGALOV, P. 1994. Research of the process for obtaining electrochemical lead alloy for restoration sliding bearings from the tractor and agricultural machinery. In 'Agricultural Machinery' magazine, vol. 6-7, 1994, pp. 34-36.

MASHKOV, Y.K. et al. 2000. Friction and modification of materials in tribosystems. Moscow : Science, 2000.

NIKOLOV, M.G. - TONCHEV, G.P - TODOROV, E. 2003. Methodology for studying the tribological characteristics of restorative vibroarc weld overlaid coating in shielding gases on cast-iron parts from the tractor and agricultural machinery. In Scientific Theses of 'Angel Kanchev' University of Ruse, vol. 40, ser. 4.1, 2003, pp. 208-212.

TONCHEV, G.P - KANGALOV, P. 2003. Testing the wearability of working surfaces of parts. Ruse : 'Angel Kanchev' University of Ruse, 2003.

TONCHEV, G.P - STANEV, L. 1983. Methodological issues of studies and control of wearability of engineering materials and parts. In Scientific Theses of 'Angel Kanchev' University of Ruse, vol. 25, ser. 2, 1983.

TONCHEV, G.P. - STANEV, S. 1979. Study of the distribution of parts of tractors YMZ-6L and MTZ-80 according to structural characteristics. In Scientific Theses of Higher Institute of Mechanical Engineering, Mechanisation and Electrification of Agriculture, vol. 21, ser. 6. Ruse : HIMEMEA, 1979.

\section{Contact address:}

Mitko Nikolov, 'Angel Kanchev' University of Ruse, Department of Repair, Reliability and ChemicalTechnologies, ul. Studentska 8, Ruse, Bulgaria, e-mail:mnikolov@uni-ruse. bg 